\title{
ARTICLE
}

\section{D3 dopamine receptors and a missense mutation of fatty acid amide hydrolase linked in mouse and men: implication for addiction}

Esmaeil Mansouri (iD ${ }^{1,2,3}$, José N. Nobrega ${ }^{2,4,5,6,7}$, Matthew N. Hill ${ }^{8}$, Rachel F. Tyndale ${ }^{4,5,6}$, Francis S. Lee ${ }^{9,10,11}$, Christian S. Hendershot ${ }^{4,5,7}$, Laura M. Best DD $^{1,3}$, Patricia Di Ciano ${ }^{4,6,12,13}$, Georgia Balsevich ${ }^{8}$, Mathew E. Sloan (iD) ${ }^{14}$, Stephen J. Kish ${ }^{2,4,5,6,12,15}$, Junchao Tong ${ }^{1,4,15,16}$, Bernard Le Foll $2,3,4,5,6,12,17$ and Isabelle Boileau ${ }^{1,2,3,4,5,15}$

The endocannabinoid and dopaminergic systems have independently been implicated in substance use disorder and obesity. We investigated a potential interaction between genetically inherited variation in fatty acid amide hydrolase $(F A A H, C 385 A)$, which metabolizes the cannabis-like endocannabinoid anandamide, and dopaminergic system, measured by dopamine receptor levels and mRNA. Binding of the dopamine D3 preferring probe [C-11]-(+)-PHNO was measured with positron emission tomography (PET) in 79 human subjects genotyped for the FAAH C385A polymorphism (36/79 AC + AA). Autoradiography with [H-3]-(+)-PHNO and in situ hybridization with a D3-specific S-35 riboprobe were carried out in 30 knock-in mice with the FAAH C385A polymorphism $(20 / 30 A C+A A)$. We found that the $F A A H$ genetic variant $C 385 A$ was associated with significantly higher $(+)-P H N O$ binding in both humans and in knock-in mice, and this effect was restricted to D3 selective brain regions (limbic striatum, globus pallidus, and ventral pallidum $(9-14 \% ; p<0.04)$ in humans and Islands of Calleja $(28 \% ; p=0.036)$ in mice). In situ hybridization with a D3-specific S-35 riboprobe in FAAH knock-in C385A mice confirmed significantly increased D3 receptor mRNA across examined regions (7-44\%; $p<0.02$ ). The association of reduced FAAH function with higher dopamine D3 receptors in human and mouse brain provide a mechanistic link between two brain systems that have been implicated in addiction-risk. This may explain the greater vulnerability for addiction and obesity in individuals with C385A genetic variant and by extension, suggest that a D3 antagonism strategy in substance use disorders should consider FAAH C385A polymorphism.

Neuropsychopharmacology (2020) 45:745-752; https://doi.org/10.1038/s41386-019-0580-8

\section{INTRODUCTION}

Endogenous cannabinoids a.k.a. endocannabinoids (anandamide or $\mathrm{N}$-arachidonoylethanolamine [1] and 2-arachidonoylglycerol or 2 -AG $[2,3])$ are lipid-based modulators of brain circuits, including the mesolimbic and corticostriatal dopamine pathways involved in reward, salience processing, and motivated behaviors [4]. These lipid transmitters are synthesized in postsynaptic neurons and act on brain circuits (including on the dopamine system) in a retrograde manner, to moderate their activity through interactions with cannabinoid receptors (CB1 and CB2). Their action is then terminated by two major enzymes: monoacylglycerol lipase [5] for 2-AG and fatty acid amide hydrolase $(F A A H)$ for anandamide. Modulating $F A A H$ enzymatic activity represents a mean of fine- tuning synaptic transmission, which can influence behaviors, including those relevant to addiction. As such, FAAH inhibitors have been proposed as a treatment strategy for multiple conditions, including substance use disorders and obesity.

Lower FAAH levels can be inherited through a genetic polymorphism prevalent in $\sim 38 \%$ of individuals of European descents [6]. This genetic polymorphism in FAAH involves the conversion of cytosine to adenosine (C385A) associated with a change in amino acid sequence at position 129 from proline to threonine (P129T). Both animal $[7,8]$ and human studies $[6,9]$ have shown that relative to CC homozygotes, individuals with C385A variant have markedly lower FAAH levels and consequentially higher anandamide [10].

\footnotetext{
${ }^{1}$ Addiction Imaging Research Group, Centre for Addiction and Mental Health, 250 College Street, Toronto, ON, Canada; ${ }^{2}$ Research Imaging Centre, Centre for Addiction and Mental Health, 250 College Street, Toronto, ON, Canada; ${ }^{3}$ Institute of Medical Sciences, University of Toronto, Toronto, ON, Canada; ${ }^{4}$ Campbell Family Mental Health Research Institute, Centre for Addiction and Mental Health, 250 College Street, Toronto, ON, Canada; ${ }^{5}$ Department of Psychiatry, University of Toronto, Toronto, ON, Canada; ${ }^{6}$ Department of Pharmacology \& Toxicology, University of Toronto, Toronto, ON, Canada; ${ }^{7}$ Department of Psychology, University of Toronto, Toronto, ON, Canada; ${ }^{8}$ The Hotchkiss Brain Institute, Departments of Cell Biology and Anatomy \& Psychiatry, University of Calgary, 3330 Hospital Drive NW, Calgary, AB T2N 4N1, Canada; ${ }^{2}$ Department of Psychiatry, Weill Cornell Medical College of Cornell University, 1300 York Avenue, New York, NY 10065, USA; ${ }^{10}$ Department of Pharmacology, Weill Cornell Medical College of Cornell University, 1300 York Avenue, New York, NY 10065, USA; ${ }^{11}$ Sackler Institute for Developmental Psychobiology, Weill Cornell Medical College of Cornell University, 1300 York Avenue, New York, NY 10065, USA; ${ }^{12}$ Translational Addiction Research Laboratory, Centre for Addiction and Mental Health, 250 College Street, Toronto, ON, Canada; ${ }^{13}$ Institute for Mental Health Policy Research, Centre for Addiction and Mental Health, Toronto, ON, Canada; ${ }^{14}$ Section on Human Psychopharmacology, Division of Intramural Clinical and Biological Research, National Institute on Alcohol Abuse and Alcoholism, Bethesda, MD 20814, USA; ${ }^{15}$ Human Brain Lab, Centre for Addiction and Mental Health, 250 College Street,

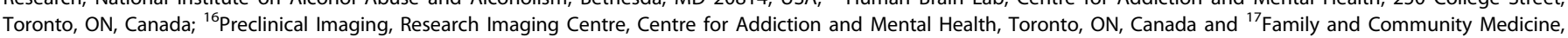
University of Toronto, Toronto, ON, Canada

Correspondence: Isabelle Boileau (isabelle.boileau@camh.ca)
}

Received: 12 October 2019 Revised: 13 November 2019 Accepted: 19 November 2019 Published online: 27 November 2019 
This polymorphism has been linked with alcohol and drug abuse, as well as with obesity $[6,8,10-14]$, though there are some conflicting findings [15-17]. Studies in healthy individuals have also shown that individuals with the FAAH C385A polymorphism have behavioral phenotypes considered risk factors for addiction, including higher reward-reactivity, impulsiveness, and higher rates of drug and alcohol use [17-19]. These human genetic studies align with preclinical investigations, which have generally shown that increasing CB1 receptor signaling, either directly using CB1 receptor agonists or partial agonist (e.g., by WIN 55,212-2, $D^{9}$ tetrahydrocannabinol (THC), anandamide, or 2-AG) or indirectly (e.g., by decreasing FAAH levels genetically or with $F A A H$ inhibitors), leads to increased pursuit of drug and non-drugrelated reward [20-23]. Consistent with these findings, decreasing CB1 receptor activity, using CB1 antagonists (SR141716A) or CB1 gene knockout animals [24], decreases motivation to seek drug and non-drug-related reward [25-27].

Functional interactions between the endocannabinoid and the dopaminergic systems are believed to contribute to the reported increase in reward sensitivity and presumably to the elevated risk for addictions [4, 28]. The dopamine system, particularly the mesolimbic striato-cortical circuits play a critical role in compulsive drug use. Using electrophysiology and microdialysis, it has been shown that $C B 1$ stimulation (with CB1 agonists (WIN 55, AM-356, JWH-018), partial agonist (THC), FAAH inhibitors (URB597) and exogenously administered anandamide, 2-AG and anandamide + URB597) increases firing activity and synaptic dopamine levels [29], and as such may promote drug seeking behavior.

Currently, despite parallel involvement of the endocannabinoid and dopamine system in addiction-related behavioral phenotypes, it is not clear whether differences in endocannabinoid metabolism by $F A A H$ affect components of the dopaminergic system in animals or in humans. As reduced $F A A H$ has been associated with behaviors that often involve the dopaminergic system, it is important to understand whether inherent variability in this enzyme is related to differences in dopaminergic system components. Currently, there has only been one in vivo study in humans, which explored the effect of the FAAH genetic polymorphism on dopaminergic receptor status [30]. This study did not show an association between the D2-preferring PET radioligand [C-11]-raclopride and $F A A H$ C385A polymorphism [30]. There is converging evidence that the dopamine D3 receptor is critically involved in the development and maintenance of addiction. The D3 dopamine receptor has been shown to be upregulated in preclinical models of substance use disorder [31] and in human psychostimulant users (both port-mortem human brain and in vivo) [31] and it has been related to addictionrelevant phenotypes (e.g., risky decision making, impulsivity). Altogether these data have raised interest in developing D3 antagonism for addiction treatment. There have been no studies investigating the dopamine D3 receptor in FAAH C385A carriers.

PET [C-11]-(+)-PHNO enables investigating D2 and D3 receptors in the living human brain [32]. Its in vivo binding in humans can be interpreted in a region-dependent manner whereby D3 vs. D2 receptor binding is, by rank order, found in substantia nigra (SN, $100 \%$ D3 selective), ventral pallidum (VP, 75\% D3 selective), globus pallidus (GP, 65\% D3 selective). In the limbic ventral striatum (LST) [C-11]-(+)-PHNO binding is mixed with $26 \%$ of its signal selective for the D3 receptor [33]. In contrast, [C-11](+)-PHNO binding in the sensory motor striatum (SMST) and associative striatum (AST) is solely attributed to D2 receptor binding [33]. Studies with D2 and D3 receptor knockout rodents have also shown that the autoradiographic binding of [H-3]$(+)$-PHNO can be interpreted in a region-dependent manner whereby the binding in VP (anterior)/Islands of Calleja is exclusively attributed to D3 receptor [34]. On the other hand, the [H-3]$(+)$-PHNO binding outside this D3-rich region in rodents is associated with D2 receptor binding [34].
In this exploratory study, a translational research approach was used to investigate the effect of FAAH C385A genetic polymorphism on (+)-PHNO radioligand binding in healthy human subjects and in FAAH C385A knock-in mice. Given the finding that D2 receptor levels are not affected by FAAH C385A [30] and the converging evidence that D3 receptor upregulation is linked with addiction-relevant phenotypes, we tentatively hypothesize that D3 but not D2 receptor binding would be elevated in FAAH C385A.

\section{MATERIALS AND METHODS}

Human subjects

All procedures were approved by the Center for Addiction and Mental Health Research Ethics Board and were conducted according to the principles expressed in the Declaration of Helsinki. Subjects were recruited from the local community in Toronto, Canada using Internet advertisements to participate in a single-PET scan study with [C-11]-(+)-PHNO. After provision of written informed consent, subjects completed a comprehensive medical/screening interview to rule out past or present significant medical conditions, neurologic illnesses or head trauma, Axis I psychiatric disorders (As per Diagnostic and Statistical Manual of Mental Disorders, fourth revision (DSM-IV) Axis I disorders [35]), magnetic resonance imaging (MRI) and PET contraindication, use of medication that may affect the central nervous system, or positive drug screening for drugs of abuse at screening and scan day.

Image acquisition and reconstruction

PET scanning was performed using either a high-resolution headdedicated PET camera system (CTI PET Systems-High Resolution Research Tomography (CPS-HRRT), Siemens Medical Imaging, Knoxville, TN) or a Siemens-Biograph HiRez XVI (Siemens Molecular Imaging, Knoxville, TN, USA) PET/CT camera system reported in Table 1. The radiosynthesis of [C-11]-(+)-PHNO and acquisition of PET images have been detailed elsewhere (Wilson et al. [32]) and included in the Supplementary Information accompanying this article.

Region of interest (ROI)-based analysis

ROI delineation and time activity curve analyses were performed using ROMI [36]. ROI-based analysis has been described elsewhere [37] and included in the Supplementary Information accompanying this article.

\section{Human $F A A H$ genotyping}

The FAAH genotype ( $r 3324420 \mathrm{C}>\mathrm{A}$ ) was determined using the Taqman SNP genotyping assay set performed on a ViiA7 thermal cycler (Life Technologies, Burlington, Ontario, Canada) with appropriate controls. Briefly $5 \mu$ l of 2x GTXpress Master mix (cat\#4401892, Life Technologies) is mixed with $10 \mathrm{ng}$ of DNA and the $40 \times$ probe (cat\#C_1897306_10, Life Technologies) in a final volume of $10 \mu \mathrm{l}$ and run for 50 cycles of $95^{\circ} \mathrm{C}$ for $1 \mathrm{~s}$ and $60^{\circ} \mathrm{C}$ for $20 \mathrm{~s}$.

\section{Generation of FAAH C385A mice}

All animal protocols were approved by the Canadian Council for Animal Care and the standards of the Animal Ethics Committee at our Institution. The introduction of C385A mutation in mice has been described in a previous publication [8]. Genetic analysis was done before and after sacrifice to confirm FAAH C385A genetic polymorphism.

In vitro [H-3]-(+)-PHNO autoradiography

Preparation of [H-3]-(+)-PHNO and autoradiography of brain tissue have been described in previous publications [34, 38]. Briefly, 30 mice genotyped for C385A genetic polymorphism (10 CC, $10 \mathrm{AC}, 10 \mathrm{AA}$ ) were sacrificed by decapitation and brains were quickly removed and frozen on dry ice. Brain tissues were then 
Table 1. Demographic characteristics of subjects.

\begin{tabular}{|c|c|c|c|c|c|}
\hline & $\begin{array}{l}\text { AA } \\
(n=5)\end{array}$ & $\begin{array}{l}\mathrm{AC} \\
(n=31)\end{array}$ & $\begin{array}{l}\mathrm{AA}+\mathrm{AC} \\
(n=36)\end{array}$ & $\begin{array}{l}\text { CC homozygotes } \\
(n=43)\end{array}$ & $\begin{array}{l}p \text {-value } \\
(\mathrm{AA}+\mathrm{AC} \text { vs. } \mathrm{CC})^{\mathrm{a}}\end{array}$ \\
\hline Age, mean \pm s.d. (range) & $43 \pm 14(21-56)$ & $42 \pm 12(21-71)$ & $42 \pm 12(21-71)$ & $38 \pm 14(20-70)$ & 0.20 \\
\hline Gender (M, F) & 2,3 & 20,11 & 22,14 & 27,16 & $\left(x^{2}=0.022\right)^{b} 0.83$ \\
\hline $\begin{array}{l}\text { Ethnicity (White, Hispanic, Asian, Black, American Indian, } \\
\text { South Asian, Egyptian, Mixed), } n\end{array}$ & $1,3,1,0,0,0,0,0$ & $24,0,1,2,0,2,2,0$ & $25,3,2,2,0,2,0,2$ & $30,1,6,2,1,2,1,0$ & $\left(x^{2}=6.888\right)^{b} 0.44$ \\
\hline Body mass index $\left(\mathrm{kg} / \mathrm{m}^{2}\right)$, mean \pm s.d. (range) & $27 \pm 5(23-36)$ & $25 \pm 4(19-34)$ & $25 \pm 4(19-36)$ & $25 \pm 3(19-31)$ & 0.38 \\
\hline Cigarette smokers, $n$ & 3 & 10 & 13 & 8 & $\left(x^{2}=2.632\right)^{b} 0.11$ \\
\hline Current alcohol use/week, mean \pm s.d. (range) & $2.90 \pm 4.22(0-10)$ & $0.98 \pm 1.95(0-8)$ & $1.25 \pm 2.39(0-10)$ & $1.28 \pm 2.39(0-12)$ & 0.95 \\
\hline HRRT vs. PET/CT, $n$ & 5,0 & 19,12 & 24,12 & 22,21 & $\left(x^{2}=2.415\right)^{\mathrm{b}} 0.12$ \\
\hline Mass injected $(\mu \mathrm{g})$, mean \pm s.d. & $7.83 \pm 1.42$ & $9.00 \pm 1.36$ & $8.84 \pm 1.41$ & $9.07 \pm 0.96$ & 0.40 \\
\hline Corrected activity $(\mathrm{mCi})$, mean \pm s.d. & $883 \pm 205$ & $1097 \pm 321$ & $1067 \pm 314$ & $1079 \pm 312$ & 0.86 \\
\hline Specific activity (mCi/ $/ \mu \mathrm{mol})$, mean \pm s.d. & $2.21 \pm 0.13$ & $2.14 \pm 0.31$ & $2.15 \pm 0.29$ & $2.19 \pm 0.31$ & 0.56 \\
\hline
\end{tabular}

stored at $-80^{\circ} \mathrm{C}$ until cryostat sectioning. Twenty-micron coronal sections were cut at -18 to $-20^{\circ} \mathrm{C}$ in a Leica cryostat and mounted onto Superfrost-plus Fisher slides. [H-3]-(+)-PHNO incubation $(2 \mathrm{nM})$; was performed in buffer containing $50 \mathrm{mM}$ Tris-HCl, $1 \mathrm{mM}$ EDTA, $1.5 \mathrm{mM} \mathrm{CaCl}, 4 \mathrm{mM} \mathrm{MgC12,} 120 \mathrm{mM} \mathrm{NaCl}$, $\mathrm{pH}=7.4$. After $2 \mathrm{~h}$ of incubation at room temperature, sections were washed in the appropriate buffer $\left(2 \times 5 \mathrm{~min}\right.$ at $4^{\circ} \mathrm{C}$ followed by a quick dip in ice cold distilled water) and left to dry at room temperature for $1 \mathrm{~h}$. Slides were then exposed to Kodak Biomax film for 6 weeks in the presence of calibrated standards (American Radiolabeled Chemicals, St. Louis, MO).

Densitometric film analyses were performed with an MCID Basic system (InterFocus Imaging, Linton, Cambridge, UK) and expressed as $\mu \mathrm{Ci} /$ gram of tissue by reference to a standard curve generated from the ${ }^{3} \mathrm{H}$-calibrated standards. Brain $\mathrm{ROls}$, as listed in Fig. 2, were defined according to the Franklin and Paxinos atlas [39]. Film analyses were performed without awareness of group membership. Note, the in vitro [H-3]-(+)-PHNO binding in VP (anterior)/Islands of Calleja is exclusively attributed to D3 receptor, whereas [H-3]-(+)-PHNO binding outside this region is associated with D2 receptor binding [34].

D3R in situ hybridization

In situ hybridization of DA D3 messenger RNA (mRNA) was performed on cryostat sections adjacent to the ones used for $[\mathrm{H}-$ 3]-(+)-PHNO autoradiography.

Following recent protocols (e.g., Creed, Hamani, \& Nobrega 2012), slides were thawed and prehybridized at room temperature. Sections were fixed in $4 \%$ paraformaldehyde for $5 \mathrm{~min}$, rinsed in $1 \times$ PBS $(2 \times 5 \mathrm{~min})$, treated with $0.1 \mathrm{M}$ triethanolamine for $5 \mathrm{~min}$, acetylated in $0.1 \mathrm{M}$ triethanolamine containing $0.25 \%$ acetic anhydride for $10 \mathrm{~min}$, and rinsed in 2x SSC. The slides were then dehydrated in graded ethanol, defatted in $100 \%$ chloroform, rehydrated, and air dried.

Hybridization of slide mounted brain sections was performed with $\left[{ }^{35} \mathrm{~S}\right]$-UTP labeled riboprobes generated by in vitro transcription using the Maxiscript kit (Ambion), and a PCR product primed by a mouse D3 receptor mRNA sequence (Genbank \# NM_007877.2, bases 429-448 and 999-980). The probe was diluted to a concentration of $18,000 \mathrm{cpm} / \mu \mathrm{l}$ in hybridization solution containing 50\% formamide, 35\% Denhardts, $10 \%$ dextran sulfate, $0.1 \times$ SSC, salmon sperm DNA $(300 \mu \mathrm{g} / \mathrm{ml})$, yeast tRNA (100 $\mu \mathrm{g} / \mathrm{ml})$, and DTT $(40 \mu \mathrm{M})$. Slides were incubated overnight at $60^{\circ} \mathrm{C}$. After hybridization, sections were rinsed with agitation using decreasing concentrations of SSC containing $25 \mathrm{~g} / \mathrm{ml}$ sodium thiosulfate. Slides were then rinsed $2 \times 20 \mathrm{~min}$ in $4 \times \mathrm{SSC}$ at $60^{\circ} \mathrm{C}$, treated in an RNase A solution $(0.5 \mathrm{M} \mathrm{NaCl}, 1 \mu \mathrm{M}$ EDTA, $10 \mu \mathrm{M}$ Tris$\mathrm{HCl}$, and RNase A $20 \mu \mathrm{g} / \mathrm{ml}$ ) at $45{ }^{\circ} \mathrm{C}$ for $40 \mathrm{~min}$, followed by $2 \times$ $24 \mathrm{~min}$ in $2 \mathrm{x}$ SSC at room temperature, $2 \times 24 \mathrm{~min}$ in $0.5 \mathrm{x}$ SSC at $60^{\circ} \mathrm{C}, 24 \mathrm{~min}$ in $0.1 \times \mathrm{SSC}$ at $60^{\circ} \mathrm{C}$, and $24 \mathrm{~min}$ in $0.1 \times \mathrm{SSC}$ at room temperature for $24 \mathrm{~min}$. Sections were then rinsed in milliQ water for $10 \mathrm{~s}$, dehydrated in $70 \%$ ethanol for $10 \mathrm{~s}$ and air dried. The slides were then exposed to Kodak BioMax film at $4{ }^{\circ} \mathrm{C}$ for 1 week.

In situ hybridization signals on film were quantified using MCID Basic 7.0 image analyses software without awareness of group membership of the samples. Densitometric data were expressed as $\mathrm{nCi} / \mathrm{gram}$ of tissue by reference to a standard curve generated from calibrated standards exposed on the same films. The same ROls (listed in Fig. 2c) from the in vitro [H-3]-(+)-PHNO autoradiography study were investigated for D3 mRNA expression.

Statistical approch

Regional (+)-PHNO binding (from both PET and autoradiography) were analyzed using general linear model (IBM SPSS Statistics 24 (Armonk, New York, USA). PET [C-11]-(+)-PHNO binding regional differences between $C C$ vs. $A A+A C$ was investigated with repeated measure analysis of variance (RM-ANOVA; 2 Groups $x 6$ ROIs). Owing to limited number of subjects with the AA genotype $(n=5)$, this group was pooled with AC subjects $(n=31)$ for the statistical analysis $(n=36)$. Autoradiography and in situ results were analyzed with univariate between-subject analysis. Sphericity was corrected using the Greenhouse-Geisser method when required. Post-hoc least significant difference pairwise comparisons were used to dissect significant interactions. Significance levels were set at 0.05 .

\section{RESULTS}

Higher [C-11]-(+)-PHNO binding in C385A human subjects [C-11]-(+)-PHNO data for these cases have been published as part of previous studies [40-46]. All healthy control subjects from previous studies who consented to pooled analysis and for whom PET and genotype data were available, were entered in the current study.

Human subjects' demographic information is reported in Table 1. A total of 79 healthy volunteers with $F A A H$ genotype and brain imaging data were included in this study. All subjects were genotyped for the FAAH C385A single-nucleotide polymorphism (SNP). Forty-three volunteers had the FAAH CC genotype and 36 had one or two copies of the A allele (31 AC and $5 \mathrm{AA}$ ). Subjects had no history of drug abuse or psychiatric disorders (as per Diagnostic and Statistical Manual of Mental 


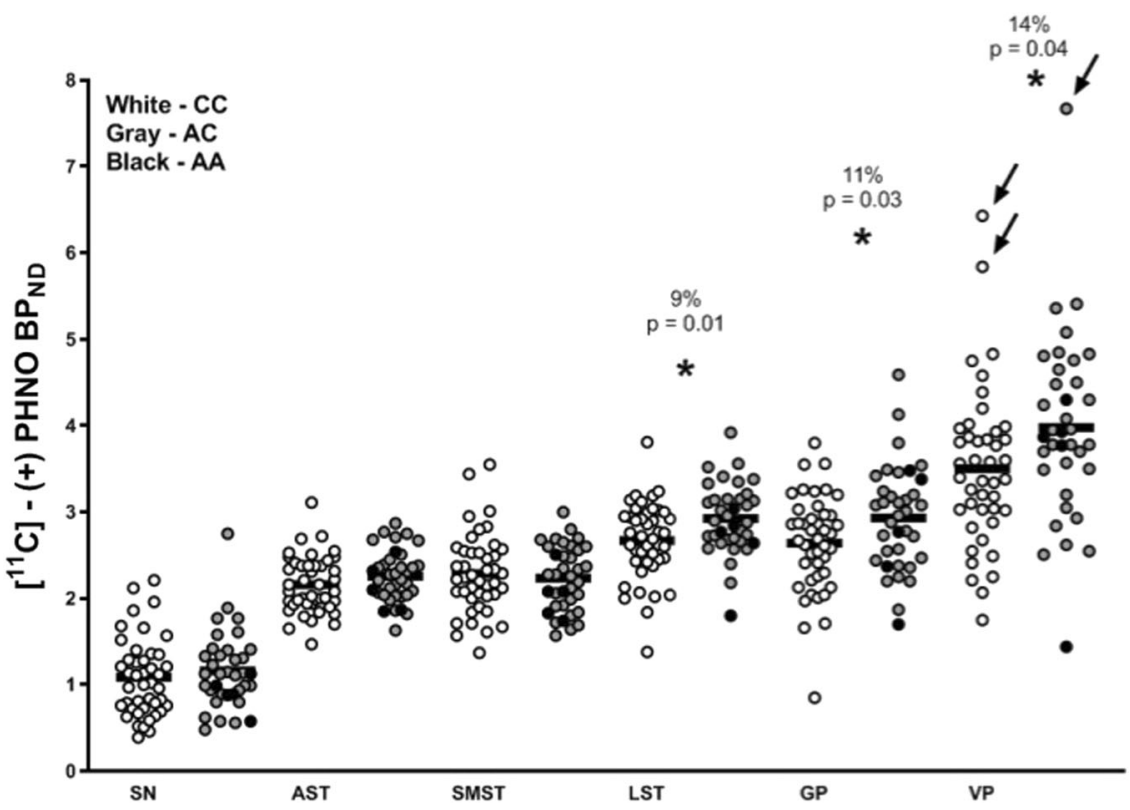

Fig. 1 [C-11]-(+)-PHNO binding in individuals with the CC ( $n=43$, white circles), AC ( $n=31$, gray circles), and AA ( $n=5$, gray circles) allele variant of the rs324420 FAAH single-nucleotide polymorphism. Removal of data from three outlier subjects with [C-11]-(+)-PHNO non-displaceable binding potential values in VP two standard deviations above the mean (arrows point to the three outliers), did not change our findings (two genotype groups $\times \mathrm{ROI}$ interaction: $F(5,360)=2.87, p=0.015$; genotype group effect: $F(1,72)=9.66, p=0.003$ ). Individuals with the AA genotype $(n=5)$ did not significantly differ from ACs. Asterisk indicates significant differences in [C-11]-(+)-PHNO nondisplaceable binding potential between AA + AC and CC genotype groups. SN substantia nigra, AST associative striatum, LST ventral limbic striatum, SMST sensory motor striatum, GP globus pallidus VP ventral pallidum.

Disorders, fourth revision (DSM-IV) Axis I disorder (First et al. [35])), did not self-report use of drugs of abuse in the 30 days prior to scanning, and tested negative for drugs of abuse on screening day as well as before their PET scan. A total of 21 subjects reported smoking cigarettes; 13 subjects with the A-variant ( 3 AAs and 10 ACs) and 8 with CC genotype (Table 1). There were no significant differences between the genotype groups in terms of age, body mass index, alcohol intake status, smoking status, [C-11]-(+)-PHNO scan parameters, and scanner type (HRRT vs. PET-CT) (Table 1).

A RM-ANOVA ( 2 genotype groups $x 6$ ROIs [SN, VP, GP, LST, AST, SMST]) indicated a significant effect of group (genotypes CC vs. $\mathrm{AA}+\mathrm{AC})$ on $[\mathrm{C}-11]-(+)-\mathrm{PHNO}$ binding $(F(1,77)=5.172, p=0.026)$ and a significant $\mathrm{ROI}$ by genotype group interaction $(F(2.48$, $190.93)=2.940, p=0.044$ ). Further RM-ANCOVA (2 genotype groups $x 6$ ROIs [SN, VP, GP, LST, AST, SMST]) with smoking status and type of PET scanning as covariates indicated a significant effect of group (genotypes CC vs. AA + AC) on [C-11]-(+)-PHNO binding $(F(1,75)=7.27, p=0.009$, overall Cohen's $d=0.71)$ and a significant $\mathrm{ROI}$ by genotype group interaction $(F(2.32,174.24)=$ $2.60, p=0.025)$. The between-group differences indicated an overall higher [C-11]-(+)-PHNO binding in the AA+AC group relative to CC group, with the greatest magnitude of effect in VP (14\%, $p=0.04$, Cohen's $d=0.48), \mathrm{GP}(11 \%, p=0.03$, Cohen's $d=$ 0.49 ), and LST (9\%, $p=0.01$, Cohen's $d=0.59$ ) (Fig. 1); nonsignificant differences were observed in the remaining D2-rich ROls $(-2$ to $4 \% ; p>0.18)$ and SN $(7 \%, p=0.47)$. Results do not survive Bonferroni correction for multiple comparisons. Subjects with AA genotype $(n=5)$ did not significantly differ from ACs ( $p>$ $0.05)$ in any ROls.

Higher in vitro [H-3]-(+)-PHNO binding in C385A knock-in mice A total of 30 adult mice (10 AA, $10 \mathrm{AC}$, and $10 \mathrm{CC}, 15 \mathrm{M}$, and $15 \mathrm{~F}$ ) were used to investigate the autoradiographic binding of $[\mathrm{H}-3]$ (+)-PHNO [34]. In order to replicate the human analyses in the mouse model, data from the mice with $A A$ and $A C$ genotypes were combined together. An univariate analysis (AA + AC vs. CC) showed significantly higher binding $(28 \%, F(1,29)=4.874, p=$ 0.036 , Cohen's $d=1.94$ ) of [H-3]-(+)-PHNO in D3-rich VP (anterior)/Islands of Calleja in C385A knock-in mice (Fig. 2a). We found no differences in other brain regions sampled (see Fig. 2a), including D2-rich ROIs (magnitude: -5 to $28 \% p>0.2$ ). [H-3]$(+)$-PHNO binding in substantia nigra compacta (SNC) $(p=0.99)$ did not differ between the genotypes, although in the mouse brain the binding in this region is not attributed to D3 binding [34]. There were no significant differences between AA vs. AC $F A A H$ genotype groups.

Higher D3 mRNA levels in in C385A knock-in mice Subsequently, to further investigate the higher levels of D3 receptors in $A A+A C$ group, in situ hybridization with a D3specific [S-35] riboprobe was carried out in the same mouse brains used for [H-3]-(+)-PHNO autoradiography (adjacent sections). A univariate analysis ( $A A+A C$ vs. $C C$ ) revealed significantly higher D3 mRNA levels in nucleus accumbens (core) $(7 \%, F(1,29)=6.274$, $p=0.018$, Cohen's $d=0.94)$, nucleus accumbens (shell) $(8 \%, F(1$, $29)=10.584, p=0.003$, Cohen's $d=1.18), V P /$ Islands of Calleja $(30 \%, F(1,29)=24.829, p=0.00003$, Cohen's $d=1.94)$, and Islands of Calleja (major) (44\%, $F(1,29)=18.546, p=0.0002$, Cohen's $d=$ 1.56) in $F A A H$ knock-in $A A+A C$ genotype mice (Fig. $2 b, c)$, suggesting that increase in [H-3]-(+)-PHNO binding in C385A mice were indeed related to upregulated $D 3$ receptors. We did not find any significant differences in D3 mRNA levels between AC and AA $F A A H$ genotypes. D3 mRNA levels in the remaining D2 ROls (olfactory tubercle, caudate-putamen (anterior pole), and lateral striatal stripe) did not show any significant differences (magnitude: $4-11 \%, p>0.1$ ) between the two groups (AA + AC vs. CC) (Fig. 2b, c).

\section{DISCUSSION}

To our knowledge this is the first study to suggest an elevation of dopamine D3 receptors in healthy human volunteers and knock-in 
a

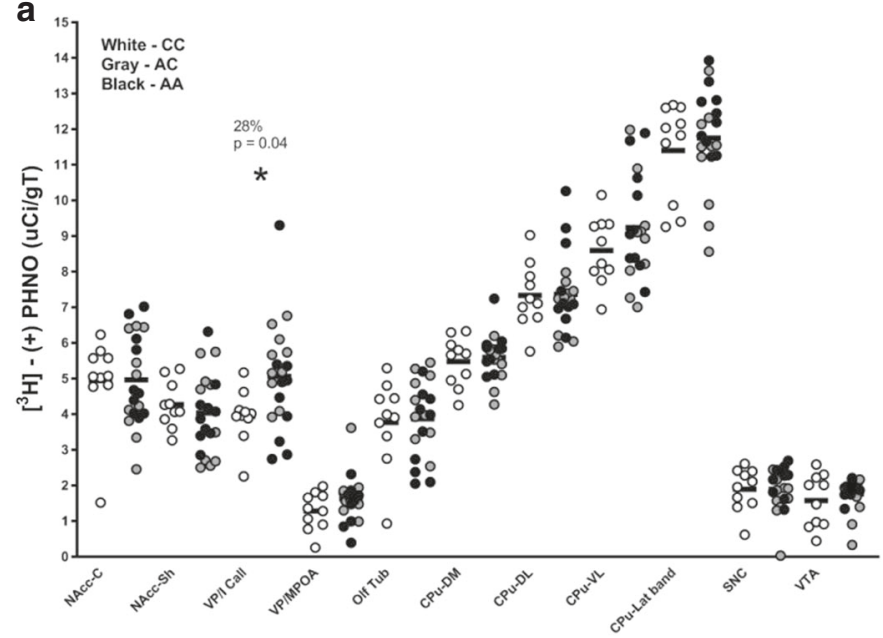

b

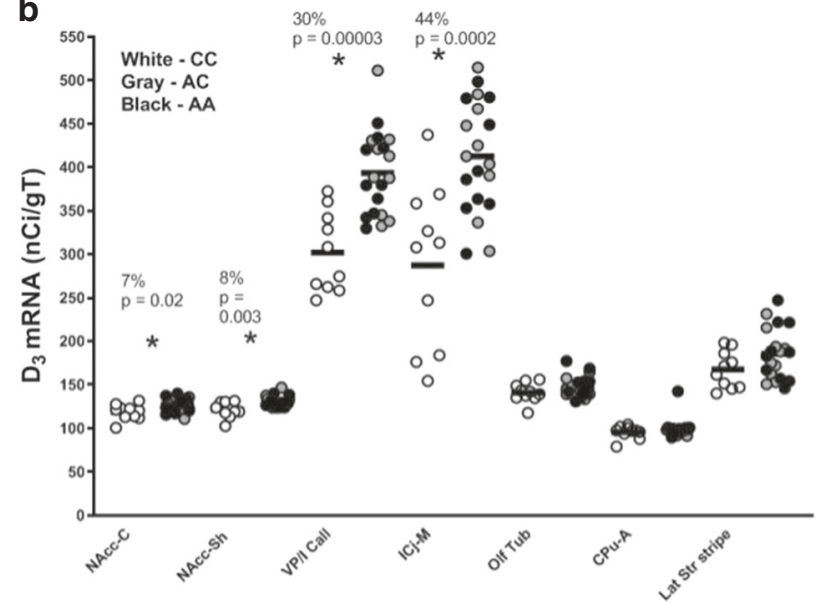

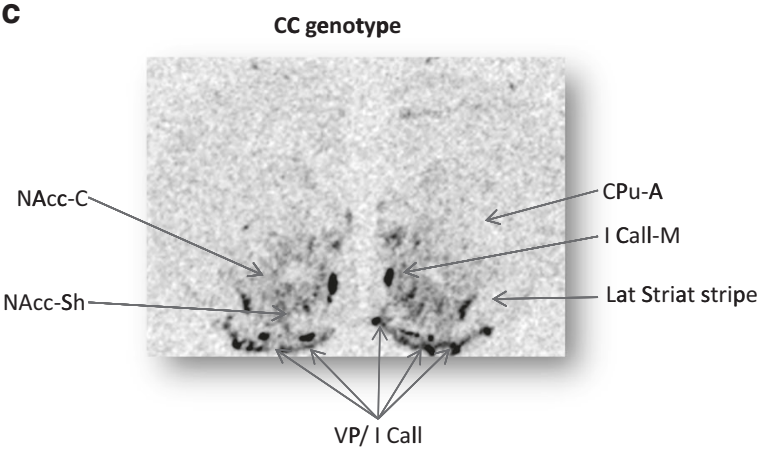
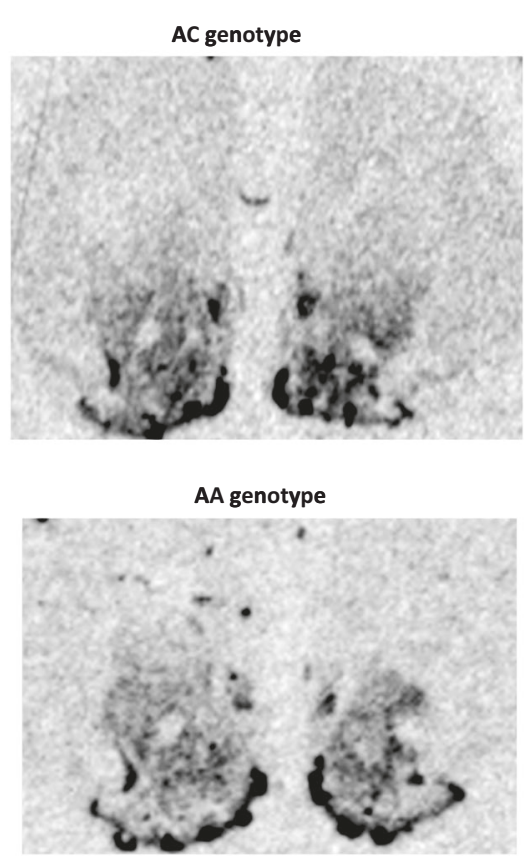

Fig. 2 [H-3]-(+)-PHNO binding and D3 mRNA levels in mice. a Comparison of mouse [H-3]-(+)-PHNO binding between CC genotypes $(n=$ 10 , white circles), AC ( $n=10$, gray circles), and AA ( $n=10$, black circles) rs324420 FAAH genotypes (AA and AC genotype groups are combined together). b Comparison of D3 mRNA levels in CC ( $n=10$, white circles), AC ( $n=10$, gray circles), and AA ( $n=10$, black circles) rs324420 FAAH genotypes. In situ hybridization was performed with a S-35-labeled probe (AA and AC genotype groups are combined together). c D3 mRNA autoradiographic images from in situ hybridization with D3-specific S-35 riboprobe from mice with CC, AC, and AA rs324420 FAAH genotypes, respectively. Asterisk indicates significant differences in [H-3]-(+)-PHNO binding or D3 mRNA levels between the two rs324420 FAAH genotype groups (CC vs. AA + AC). There were no significant differences between AA and AC groups. NAcc-C nucleus accumbens Core, NAcc-Sh nucleus accumbens shell, VP/I Call ventral pallidum (anterior)/Islands of Calleja, VP/MPOA ventral palidum (medial)/medial preoptic area, ICj-M Islands of Calleja (major), Olf Tub olfactory tubercle, CPu-DM Caudate-putamen (dorsomedial), CPu-DL Caudate-putamen (dorsolateral), CPu-VL Caudate-putamen (ventrolateral), CPu-Lat band Caudate-putamen (exterior lateral band), SNC Substantia nigra compacta, VTA ventral tegmental area, CPu-A Caudate-putamen (anterior pole), Lat Str stripe lateral striatal stripe.

mice with reduced $F A A H$ function. We found that individuals with the $A$-variant $(A C+A A)$, which we have previously shown to have lower brain binding of the FAAH PET probe [C-11]CURB (see Boileau et al. [9]), have significantly higher PET [C-11]-(+)-PHNO binding specifically in D3-rich brain regions, including the LST, GP, and VP in human; with no differences in SN and D2-specific striatal regions. Despite some [C-11]-(+)-PHNO D2 receptor binding in GP, VP, and LST, our finding in human, which was robustly replicated using $[\mathrm{H}-3]-(+)-\mathrm{PHNO}$ binding and D3 receptor mRNA in FAAH C385A knock-in mice, may reflect selective D3 but not D2 receptor upregulation. This is in-line with a previous report of no differences in D2 receptor levels between the two $F A A H$ genotypes as measured by the non-specific dopamine D2 receptor probe [C-11]-raclopride [30].

The D3 receptor differs from the D2 (and D1) in terms of transduction system, pharmacology, and importantly brain localization selective to the ventral striatum, Islands of Calleja, septum, and nucleus basalis [47]. The D3 dopamine receptor became a main focus of research in the addiction field because of this selective anatomical distribution in brain, which overlaps with key neurocircuits that underlie processes believed to be aberrant in addiction (e.g., motivation, inhibitory control, emotion, and learning) [47]. Interestingly preclinical and neuroimaging studies in humans have also suggested that, unlike the D2 receptor, which is downregulated in addiction, the D3 receptor is paradoxically upregulated in addiction to stimulants (Boileau et al. [44] and Payer et al. [48]) and is related to addiction behavioral phenotypes.

The exact mechanisms potentially leading to a selective D3 upregulation in humans and mice with the FAAH C385A variant are currently unknown. Upregulation of the D3 receptor in the striatum has been shown to be dependent on dopamine stimulation of the D1/5 receptor and release of the brain derived neurotrophic factor (BDNF) from corticostriatal neurons [49]. One possibility is that having inherently lower $F A A H$ results in higher levels of brain anandamide and/or other $F A A H$ substrates (i.e., 
oleylethanolamide (OEA), palmitylethanolamide (PEA)), which may elevate mesolimbic dopamine (through CB1 and / or transient receptor potential vanilloid receptor 1 (TRPV1) or peroxisomeproliferator activated receptor (PPAR)-a routes), leading to greater activation of D1/5 receptors (and greater BDNF release). There are currently no studies to support this as basic investigations of dopamine system status in FAAH knockout mice at baseline have not been conducted.

Acute exogenous administration of intravenous anandamide or methanandamide [28, 50], OEA, and PEA [51], as well as administration of a FAAH inhibitor (URB597), which elevate anandamide (as well as OEA and PEA), increases dopamine dialysates [51, 52], and nicotine-induced dopamine release in some [53] though not all studies [54]. Evidence for increased dopamine cell firing and extracellular dopamine levels also comes from studies of CB1 stimulation by exogenous cannabinoids such as THC and its analogs (e.g., WIN55212-2) [29]. Indeed, autoradiographic studies with [H-3]-(+)-PHNO in rodents, undergoing chronic THC exposure have shown an upregulation of D3 receptors in nucleus accumbens and ventral pallidum $[55,56]$.

Finally, given that BDNF is required to increase expression of D3 receptors, one could speculate that deficiencies in $F A A H$ (or increases in $F A A H$ substrates) may be associated with increases in BDNF. Indeed, some studies have also shown that stimulation of the $\mathrm{CB} 1$ receptor (for example by THC) and inhibition of FAAH induced the release of neurotrophins such as BDNF [57-60]. Furthermore, PPAR- $a$ agonists (PEA, Gemfibrozil, WY-14643, and Fenofibrate) have been shown to restore BDNF signaling in animal models of chronic unpredictable mild stress and autism spectrum disorders [61-64]. Studies of D3 receptor expression and BDNF should be carried out in knock-in mice with the FAAH C385A variant similar to the human genetic variant.

We did not find a significant difference in [C-11]-(+)-PHNO binding between the two groups in $\mathrm{SN}$, the brain region that reflects exclusive [C-11]-(+)-PHNO to D3 binding in the human. This could be explained, in part, by the fact that more than half of our PET scans (45 out of 79 scans) were done on PET/CT camera system, which generates a lower resolution signal and "noisier" measurements compared to HRRT scans. Nonetheless statistical analyses of the HRRT subgroup, which may be underpowered, did not yield any significant differences in SN. Another possibility is that D3 upregulation may not occur in dopamine cell body of healthy controls with the FAAH C385A variant. Although our PET studies in methamphetamine and cocaine users have shown increased [C-11]-(+)-PHNO binding in SN (and to a lesser extend in VP and GP) [44, 48]; upregulation of D3 binding in animals exposed to dopamine elevating drugs has not in fact been reported in SN [31], raising the possibility that increases in [C-11]$(+)$-PHNO binding in SN of stimulant users may be driven by low levels of DA [31]. The regional D3 "upregulation" in the current study also differs from the pattern observed in stimulant addiction, in that an elevation in [C-11]-(+)-PHNO binding in LST is observed in the current study. Failure to find an elevation in [C11]-(+)-PHNO binding in LST in stimulant users may be due to competing decreases in D2 receptor levels [31].

This study is not without limitations. For one we did not obtain mRNA levels for the D2 receptor for comparison and therefore cannot entirely rule out the possibility that an elevation in D2 receptor in mixed D2/3 regions (e.g., LST) could contribute to the findings. It is however unlikely that the elevation in (+)-PHNO binding in both mouse and human results from increased D2 receptors based on the regional pattern of the effect, in which no differences in D2 selective regions were found. In this regard both [C-11]-(+)-PHNO occupancy study as well as knockout studies in animal have shown that the regions in which (+)-PHNO is elevated in the mouse study, are selective for D3. Secondly, despite the fact that the study was conducted in a large sample of well-characterized healthy controls, the sample is still considered small for a genetic polymorphism investigation and the differences in [C-11]-(+)-PHNO binding observed were relatively small given the high test-retest variability in these regions ( 20\%) [65]. Furthermore, this study was a retrospective study conducted on two scanners over a long period of time (2005-2017). We were not able to amass behavioral information of traits relevant to addiction. Future studies will have to investigate whether low$F A A H$ and high-D3 dopamine may be related to behavioral constructs such as cognitive and motor impulsivity and reward sensitivity, which have been linked to dopamine and $F A A H$ independently.

Previous studies found age-dependent adaptive changes in endocannabinoid metabolism in mice and rats [66,67]. Although not significant, the $A C+A A$ human group was on average older than CC FAAH genotype group. However, the inclusion of age as a covariate in the analysis did not change the presented outcome. Even though, we did not find an effect of gender between the genotype groups, previous studies have shown sex-linked endocannabinoid system differences in rodents $[68,69]$. This could be related to the limited power in our samples, although in our own studies with PET radioligand [C-11]CURB, we did not find any significant sex-linked differences (unpublished data).

In conclusion, we report that a common $F A A H$ genetic polymorphism selectively affects dopamine D3 receptor levels. These results may implicate a dopaminergic (upregulated D3) mechanism in elevated risk for addiction and obesity in individuals with the FAAH C385A variant.

\section{FUNDING AND DISCLOSURE}

We acknowledge these sources of funding: Canada Research Chair in Pharmacogenomics (RFT); Canadian Institutes of Health Research (CIHR) grant BSB-389329 and BSB-389342 and FDN154294 (RFT); the Campbell Family Mental Health Research Institute of the Center for Addiction and Mental Health (CAMH).

Esmaeil Mansouri declares no conflict of interest. José $\mathrm{N}$. Nobrega declares no conflict of interest. Matthew N. Hill declares no conflict of interest. Rachel F. Tyndale has consulted for Quinn Emmanual, Apotex, and Ethismos on unrelated topics. Francis $\mathrm{S}$. Lee declares no conflict of interest. Christian S. Hendershot declares no conflict of interest. Laura M. Best declares no conflict of interest. Patricia Di Ciano declares no conflict of interest. Georgia Balsevich declares no conflict of interest. Mathew E. Sloan declares no conflict of interest. Stephen J. Kish declares no conflict of interest. Junchao Tong declares no conflict of interest. Bernard Le Foll declares no conflict of interest. Isabelle Boileau declares no conflict of interest.

\section{ACKNOWLEDGEMENTS}

We thank Roger Raymond and Meagan Billyard for help with the autoradiography analyses.

\section{ADDITIONAL INFORMATION}

Supplementary Information accompanies this paper at https://doi.org/10.1038/ s41386-019-0580-8.

Publisher's note Springer Nature remains neutral with regard to jurisdictional claims in published maps and institutional affiliations.

\section{REFERENCES}

1. Devane WA, et al. Isolation and structure of a brain constituent that binds to the cannabinoid receptor. Science. 1992;258:1946-9.

2. Mechoulam R, et al. Identification of an endogenous 2-monoglyceride, present in canine gut, that binds to cannabinoid receptors. Biochem Pharm. 1995;50:83-90.

3. Sugiura T, et al. 2-Arachidonoylglycerol: a possible endogenous cannabinoid receptor ligand in brain. Biochem Biophys Res Commun. 1995;215:89-97. 
4. Parsons LH, Hurd YL. Endocannabinoid signalling in reward and addiction. Nat Rev Neurosci. 2015;16:579-94.

5. Dinh TP, et al. Brain monoglyceride lipase participating in endocannabinoid inactivation. Proc Natl Acad Sci USA. 2002;99:10819-24.

6. Sipe JC, et al. A missense mutation in human fatty acid amide hydrolase associated with problem drug use. Proc Natl Acad Sci USA. 2002;99:8394-9.

7. Chiang KP, et al. Reduced cellular expression and activity of the P129T mutant of human fatty acid amide hydrolase: evidence for a link between defects in the endocannabinoid system and problem drug use. Hum Mol Genet. 2004;13:2113-9.

8. Dincheva I, et al. FAAH genetic variation enhances fronto-amygdala function in mouse and human. Nat Commun. 2015;6:6395.

9. Boileau I, et al. The fatty acid amide hydrolase C385A variant affects brain binding of the positron emission tomography tracer [11C]CURB. J Cereb Blood Flow Metab. 2015;35:1237-40.

10. Sipe JC, et al. Biomarkers of endocannabinoid system activation in severe obesity. PLoS ONE. 2010;5:e8792.

11. Flanagan JM, et al. The fatty acid amide hydrolase 385 A/A (P129T) variant: haplotype analysis of an ancient missense mutation and validation of risk for drug addiction. Hum Genet. 2006;120:581-8.

12. Tyndale RF, et al. The fatty acid amide hydrolase C385A (P129T) missense variant in cannabis users: studies of drug use and dependence in Caucasians. Am J Med Genet B Neuropsychiatr Genet. 2007;144B:660-6.

13. Sipe JC, et al. Overweight and obesity associated with a missense polymorphism in fatty acid amide hydrolase (FAAH). Int J Obes (Lond). 2005;29:755-9.

14. Monteleone $\mathrm{P}$, et al. The cDNA $385 \mathrm{C}$ to $\mathrm{A}$ missense polymorphism of the endocannabinoid degrading enzyme fatty acid amide hydrolase (FAAH) is associated with overweight/obesity but not with binge eating disorder in overweight/obese women. Psychoneuroendocrinology. 2008;33:546-50.

15. Morita $\mathrm{Y}$, et al. A nonsynonymous polymorphism in the human fatty acid amide hydrolase gene did not associate with either methamphetamine dependence or schizophrenia. Neurosci Lett. 2005;376:182-7.

16. Iwasaki $\mathrm{S}$, et al. Association study between alcoholism and endocannabinoid metabolic enzyme genes encoding fatty acid amide hydrolase and monoglyceride lipase in a Japanese population. Psychiatr Genet. 2007;17:215-20.

17. Buhler KM, et al. Risky alcohol consumption in young people is associated with the fatty acid amide hydrolase gene polymorphism C385A and affective rating of drug pictures. Mol Genet Genomics. 2014;289:279-89.

18. Hariri AR, et al. Divergent effects of genetic variation in endocannabinoid signaling on human threat- and reward-related brain function. Biol Psychiatry. 2009;66:9-16.

19. Schacht JP, Selling RE, Hutchison KE. Intermediate cannabis dependence phenotypes and the FAAH C385A variant: an exploratory analysis. Psychopharmacol (Berl). 2009;203:511-7.

20. Caille $S$, et al. Specific alterations of extracellular endocannabinoid levels in the nucleus accumbens by ethanol, heroin, and cocaine self-administration. J Neurosci. 2007;27:3695-702.

21. Simonnet $A$, Cador $M$, Caille $S$. Nicotine reinforcement is reduced by cannabinoid CB1 receptor blockade in the ventral tegmental area. Addict Biol. 2013;18:930-6.

22. Caille S, Parsons LH. Cannabinoid modulation of opiate reinforcement through the ventral striatopallidal pathway. Neuropsychopharmacology. 2006;31:804-13.

23. Jarbe TU, Liu Q, Makriyannis A. Antagonism of discriminative stimulus effects of delta(9)-THC and (R)-methanandamide in rats. Psychopharmacol (Berl). 2006;184:36-45.

24. Ledent $C$, et al. Unresponsiveness to cannabinoids and reduced addictive effects of opiates in CB1 receptor knockout mice. Science. 1999;283:401-4.

25. Soria G, et al. Lack of $C B 1$ cannabinoid receptor impairs cocaine selfadministration. Neuropsychopharmacology. 2005;30:1670-80.

26. Delis $F$, et al. Attenuation of cocaine-induced conditioned place preference and motor activity via cannabinoid CB2 receptor agonism and CB1 receptor antagonism in rats. Int J Neuropsychopharmacol. 2017;20:269-78.

27. Ward SJ, Dykstra LA. The role of $C B 1$ receptors in sweet versus fat reinforcement: effect of CB1 receptor deletion, CB1 receptor antagonism (SR141716A) and CB1 receptor agonism (CP-55940). Behav Pharm. 2005;16:381-8.

28. Solinas M, Goldberg SR, Piomelli D. The endocannabinoid system in brain reward processes. Br J Pharm. 2008;154:369-83.

29. Oleson EB, JF Cheer. A brain on cannabinoids: the role of dopamine release in reward seeking. Cold Spring Harb Perspect Med. 2012; 2:a012229.

30. Pecina $M$, et al. FAAH selectively influences placebo effects. Mol Psychiatry. 2014;19:385-91.

31. Payer D, Balasubramaniam G, Boileau I. What is the role of the D3 receptor in addiction? A mini review of PET studies with [(11)C]-(+)-PHNO. Prog Neuropsychopharmacol Biol Psychiatry. 2014;52:4-8.

32. Wilson $A A$, et al. Radiosynthesis and evaluation of [11C]-(+)-4-propyl$3,4,4 a, 5,6,10 \mathrm{~b}$-hexahydro-2H-naphtho[1,2-b][1,4]oxazin-9-ol as a potential radiotracer for in vivo imaging of the dopamine D2 high-affinity state with positron emission tomography. J Med Chem. 2005;48:4153-60.

33. Tziortzi AC, et al. Imaging dopamine receptors in humans with [11C]-(+)-PHNO dissection of D3 signal and anatomy. Neuroimage. 2011;54:264-77.

34. Nobrega JN, Seeman P. Dopamine D2 receptors mapped in rat brain with $[3 \mathrm{H}](+)$ PHNO. Synapse. 1994;17:167-72.

35. First MB, et al. Structured clinical interview for DSM-IV axis I disorders-patient edition (SCID-IP, version 2.0). New York: Biometrics Research Department, New York State Psychiatric Institute; 1996.

36. Rusjan $P$, et al. An automated method for the extraction of regional data from PET images. Psychiatry Res. 2006;147:79-89.

37. Boileau I, et al. Decreased binding of the D3 dopamine receptor-preferring ligand [11C]-(+)-PHNO in drug-naive Parkinson's disease. Brain. 2009;132(Pt 5):1366-75.

38. Seeman $P$, et al. Dopamine receptors labelled by PHNO. Synapse. 1993;14:254-62

39. Franklin K, Paxinos G. The mouse brain in stereotaxic coordinates. San Diego, CA: Academic Press; 1977.

40. Di Ciano $P$, et al. Influence of nicotine metabolism ratio on [11C]-(+)-PHNO PET binding in tobacco smokers. Int J Neuropsychopharmacol. 2018;21:503-12.

41. Malik $S$, et al. Deep TMS of the insula using the H-coil modulates dopamine release: a crossover $[(11) \mathrm{C}]$ PHNO-PET pilot trial in healthy humans. Brain Imaging Behav. 2017;5:1306-17.

42. Payer $D$, et al. Corticotropin-releasing hormone and dopamine release in healthy individuals. Psychoneuroendocrinology. 2017;76:192-6.

43. Payer DE, et al. D3 dopamine receptor-preferring [11C]PHNO PET imaging in Parkinson patients with dyskinesia. Neurology. 2016;86:224-30.

44. Boileau I, et al. Higher binding of the dopamine D3 receptor-preferring ligand [11C]-(+)-propyl-hexahydro-naphtho-oxazin in methamphetamine polydrug users: a positron emission tomography study. J Neurosci. 2012;32:1353-9.

45. Le Foll B, et al. Occupancy of dopamine D3 and D2 receptors by buspirone: A [11C] (+)-PHNO PET study in humans. Neuropsychopharmacology. 2016;41:529-37.

46. Di Ciano $P$, et al. Occupancy of dopamine D2 and D3 receptors by a novel D3 partial agonist BP1.4979: a $[(11) \mathrm{C}]-(+)-\mathrm{PHNO}$ PET study in humans. Neuropsychopharmacology. 2019;44:1284-90.

47. Bouthenet $M L$, et al. Localization of dopamine $D 3$ receptor $m R N A$ in the rat brain using in situ hybridization histochemistry: comparison with dopamine D2 receptor mRNA. Brain Res. 1991;564:203-19.

48. Payer $\mathrm{DE}$, et al. Heightened $\mathrm{D} 3$ dopamine receptor levels in cocaine dependence and contributions to the addiction behavioral phenotype: a positron emission tomography study with [11C]-+-PHNO. Neuropsychopharmacology. 2014;39:311-8.

49. Guillin O, et al. BDNF controls dopamine D3 receptor expression and triggers behavioural sensitization. Nature. 2001;411:86-9.

50. Solinas $M$, et al. Anandamide administration alone and after inhibition of fatty acid amide hydrolase (FAAH) increases dopamine levels in the nucleus accumbens shell in rats. J Neurochem. 2006;98:408-19.

51. Murillo-Rodriguez E, et al. Administration of URB597, oleoylethanolamide or palmitoylethanolamide increases waking and dopamine in rats. PLoS ONE. 2011;6:e20766.

52. Murillo-Rodriguez $\mathrm{E}$, et al. Effects of the fatty acid amide hydrolase inhibitor URB597 on the sleep-wake cycle, c-Fos expression and dopamine levels of the rat. Eur J Pharm. 2007;562:82-91.

53. Pavon FJ, et al. Fatty acid amide hydrolase (FAAH) inactivation confers enhanced sensitivity to nicotine-induced dopamine release in the mouse nucleus accumbens. Addict Biol. 2018;23:723-34.

54. Mascia $P$, et al. Blockade of nicotine reward and reinstatement by activation of alpha-type peroxisome proliferator-activated receptors. Biol Psychiatry. 2011;69:633-41.

55. Ginovart N, et al. Chronic Delta(9)-tetrahydrocannabinol exposure induces a sensitization of dopamine $\mathrm{D}(2) /(3)$ receptors in the mesoaccumbens and nigrostriatal systems. Neuropsychopharmacology. 2012;37:2355-67.

56. Tournier BB, et al. Time-dependent effects of repeated THC treatment on dopamine D2/3 receptor-mediated signalling in midbrain and striatum. Behav Brain Res. 2016;311:322-9.

57. Derkinderen $P$, et al. Regulation of extracellular signal-regulated kinase by cannabinoids in hippocampus. J Neurosci. 2003;23:2371-82.

58. Heyman $E$, et al. Intense exercise increases circulating endocannabinoid and BDNF levels in humans-possible implications for reward and depression. Psychoneuroendocrinology. 2012;37:844-51.

59. Vinod $K Y$, et al. Dysfunction in fatty acid amide hydrolase is associated with depressive-like behavior in Wistar Kyoto rats. PLoS ONE. 2012;7:e36743.

60. Khaspekov LG, et al. Involvement of brain-derived neurotrophic factor in cannabinoid receptor-dependent protection against excitotoxicity. Eur J Neurosci. 2004;19:1691-8.

61. Cristiano $C$, et al. Palmitoylethanolamide counteracts autistic-like behaviours in BTBR T tf/J mice: Contribution of central and peripheral mechanisms. Brain, Behavior, and Immunity. 2018;74:166-75. 
D3 dopamine receptors and a missense mutation of fatty acid amide...

E Mansouri et al.

752

62. Ni YF, et al. Gemfibrozil has antidepressant effects in mice: Involvement of the hippocampal brain-derived neurotrophic factor system. J Psychopharmacol. 2018;32:469-81.

63. Yang R, et al. WY-14643, a selective agonist of peroxisome proliferator-activated receptor-alpha, ameliorates lipopolysaccharide-induced depressive-like behaviors by preventing neuroinflammation and oxido-nitrosative stress in mice. Pharm Biochem Behav. 2017;153:97-104.

64. Jiang B, et al. Antidepressant-like effects of fenofibrate in mice via the hippocampal brain-derived neurotrophic factor signalling pathway. Br J Pharm. 2017;174:177-94.

65. Willeit $M$, et al. First human evidence of $d$-amphetamine induced displacement of a D2/3 agonist radioligand: A [11C]-(+)-PHNO positron emission tomography study. Neuropsychopharmacology. 2008;33:279-89.
66. Maccarrone $M$, et al. Age-related changes of anandamide metabolism in CB1 cannabinoid receptor knockout mice: correlation with behaviour. Eur J Neurosci. 2002;15:1178-86.

67. Pascual AC, et al. Normal aging in rats and pathological aging in human Alzheimer's disease decrease FAAH activity: modulation by cannabinoid agonists. Exp Gerontol. 2014;60:92-9.

68. Basavarajappa BS, et al. Increased ethanol consumption and preference and decreased ethanol sensitivity in female FAAH knockout mice. Neuropharmacology. 2006;50:834-44.

69. Hlavacova $\mathrm{N}$, et al. Inhibition of fatty-acid amide hydrolyse (FAAH) exerts cognitive improvements in male but not female rats. Endocr Regul. 2015;49:131-6. 